\title{
Observations of 44 extragalactic radio sources with the VLBA at $92 \mathrm{~cm}$
}

\section{A list of potential calibrators and targets for LOFAR and RadioAstron}

\author{
H. Rampadarath ${ }^{1,2}$, M. A. Garrett ${ }^{3,2,4}$, and A. Polatidis ${ }^{1,3}$ \\ 1 Joint Institute for VLBI in Europe (JIVE), Postbus 2, 7990 AA Dwingeloo, The Netherlands \\ e-mail: [rampadarath; polatidis]@astron.nl \\ 2 Sterrewacht Leiden, Leiden University, Oort Building, Neils Bhorweg 2, 2333 CA, Leiden, The Netherlands \\ 3 Netherlands Institute for Radio Astronomy (ASTRON), Postbus 2, 7990 AA Dwingeloo, The Netherlands \\ e-mail: garrett@astron.nl \\ 4 Centre for Supercomputing, Swinburne University of Technology, Mail number H39, PO Box 218, Hawthorn, \\ Victoria 3122, Australia
}

Received 5 February 2009 / Accepted 14 March 2009

\section{ABSTRACT}

\begin{abstract}
Aims. We have analysed VLBA $92 \mathrm{~cm}$ archive data of 44 extragalactic sources in order to identify early targets and potential calibrator sources for the LOFAR radio telescope and the RadioAstron space VLBI mission. Some of these sources will also be suitable as "inbeam" calibrators, permitting deep, wide-field studies of other faint sources in the same field of view.

Methods. All publicly available VLBA $92 \mathrm{~cm}$ data observed between 1 January 2003 to December 31, 2006 have been analysed via an automatic pipeline, implemented within AIPS. The vast majority of the data are unpublished.

Results. The sample consists of 44 sources, 34 of which have been detected on at least one VLBA baseline. 30 sources have sufficient data to be successfully imaged. Most of the sources are compact, with a few showing extended structures. Of the 30 sources imaged, 13 are detected on the longest VLBA baselines $(\sim 9 \mathrm{M} \lambda)$, while all were detected on baselines greater than $2 \mathrm{M} \lambda$ (the maximum baseline of LOFAR including the current international baselines).
\end{abstract}

Key words. radiation mechanisms: general - radio continuum: general - techniques: interferometric

\section{Introduction}

Low-frequency observations of the radio sky are set to be transformed by a new generation of radio telescopes. In particular, instruments such as LOFAR (the Low Frequency Array) will permit large areas of the sky to be surveyed at very low-frequencies (30-230 MHz) with unprecedented sensitivity, and with arcsecond and sub-arcsecond resolution. The base LOFAR configuration foresees at least 36 stations being distributed across the Netherlands with at least 9 additional international stations located in Germany (5), Sweden (1), France (1), the UK (1) and Italy (1). Further stations are planned in other European countries, including Poland, Austria, Ireland and the Ukraine (Garrett et al. 2009). Initial science observations with LOFAR are expected to begin in 2009.

The extension of LOFAR to baseline lengths of a few thousand kilometres presents several new challenges. With a resolution of up to $\sim 0.2$ arcsec, the sky density of suitable calibrator sources may be a significant issue especially at the lowest frequencies. In particular, very little is known about the morphology of radio sources at these low frequencies and high resolutions. A very similar situation exists with the space VLBI mission RadioAstron, due to be launched in October 2009 (Zakharov 2007). With a maximum baseline length of $350000 \mathrm{~km}$, RadioAstron will have a fringe spacing of 0.540 milliarcsec at $327 \mathrm{MHz}$ (see the RadioAstron booklet for more details ${ }^{1}$ ).

Altschuler et al. (1995) presented the results of a global Mark II VLBI $92 \mathrm{~cm}$ snapshot survey. Their sample contained 16 sources, of which there were 3 radio galaxies: $0116+319$ (4C 31.04), 1117+146 (4C 14.41), 2050+364 (DA529); 4 BL Lac objects: 0235+164, 0723008, 0735+178, 0851+202 (OJ287); and 9 quasars: $0333+321$ (NRAO140), 1055+018 (4C 01.28), 1422+202, 1611+343 (DA406), 1633+382 (4C 38.41), 1901+319 (3C 395), 2145+067 (4C 06.69), 2230+114 (CTA102), 2251+158 (3C 454.3). All of which were resolved on baselines longer than 2-6 M $\lambda$. Only 2 of the sources observed by altetal95 (3C 395 and 3C 454.3) are part of the sample presented in this paper.

Chuprikov et al. (1999) have presented a list of 61 sources, observed in two separate experiments, at $327 \mathrm{MHz}$ with a global Mark II VLBI array. Of the 61 sources observed, 25 were recommended for further detailed observations and images were made of 2 of these sources. 3 of the sources we present in this paper (3C 273, 3C 345 and BL Lac), are also included in Chuprikov's sample.

Lenc et al. (2008) have conducted a deep wide field VLBI survey of the sky at $92 \mathrm{~cm}$ with a global VLBI array using two in-beam calibrators. Of the 272 potentially detectable targets, about 20 sources show compact, milliarcsecond scale structures. These results are encouraging, and suggest that deep, wide-field

\footnotetext{
${ }^{1}$ http://www.asc.rssi.ru/radioastron
} 
Table 1. 327 MHz VLBA archived projects from 2003 to 2006.

\begin{tabular}{|c|c|c|}
\hline Project & $\begin{array}{c}\text { Date of } \\
\text { Observation }\end{array}$ & Common Source name \\
\hline BM201B & $16 / 07 / 03$ & $3 \mathrm{C} 454.3, \mathrm{~J} 2327+0940, \mathrm{~J} 2334+0736, \mathrm{~J} 2336+2828$ \\
\hline BM193 PASS1 & $30 / 08 / 03$ & NGC 7674, J2330+1100, 3C 454.3 \\
\hline BM193 PASS2 & $30 / 08 / 03$ & $\mathrm{~J} 2322+081, \mathrm{~J} 2330+11,3 \mathrm{C} 454.3$ \\
\hline BC138A PASS1 & 09/08/03 & $\mathrm{J} 1331+3030,3 \mathrm{C} 336, \mathrm{~J} 1816+3457$ \\
\hline BC138A PASS2 & 09/08/03 & $\mathrm{J} 1331+3030, \mathrm{~J} 1619+2247, \mathrm{~J} 1816+3457$ \\
\hline BEO32A PASS1 & 09/11/03 & 3C 286, 3C 395, 3C 418, 3C 454.3, BLLAC, Cygnus-A, J1821+3945, J1829+4844, J1924+3329, J2007+4029 \\
\hline BEO32A PASS2 & 09/11/03 & 3C 286, 3C 395, 3C 418, 3C 454.3, BLLAC, J2007+4029, J1821+3945, J1829+4844, J1924+3329, J2007+4029 \\
\hline BWO67Q & $31 / 12 / 03$ & 3C 120, 3C 286, 3C 147, J0423-0120 \\
\hline $\mathrm{BC} 138 \mathrm{C}$ & $30 / 01 / 04$ & 3C 273, 3C 275.1, J1331+3030 \\
\hline BH116 & $11 / 03 / 04$ & 3C 286; J1512-0905 \\
\hline BJ046R & $23 / 04 / 04$ & J1150-0024, 3C 147, 3C 274 \\
\hline BB197 & 08/07/04 & 3C 454.3, J2022+6136, J1829+4844 \\
\hline BH126A & $23 / 12 / 04$ & 3C $147, \mathrm{~J} 1150-002$ \\
\hline BC150 & $11 / 02 / 05$ & $\mathrm{~J} 1746+6226,3 \mathrm{C} 286,3 \mathrm{C} 345$ \\
\hline ВT070B & $13 / 06 / 05$ & $\mathrm{~J} 0405+3803,3 \mathrm{C} 111,3 \mathrm{C} 84$ \\
\hline BFO87 PASS1 & $03 / 12 / 05$ & J2005+7752, J0955+6940 \\
\hline BF087 PASS2 & $03 / 12 / 05$ & M 82, J2005+7752 \\
\hline BH135D & $06 / 02 / 06$ & J0955+6940, J1150-002, 3C 147, 3C 274 \\
\hline BK131C & 08/06/06 & J1230-1139, J1333+1649, 3C 286, 3C 345, J0539-283 \\
\hline BK131A & 09/06/06 & J0440-4333, J0204+3649, J0319+4130, J2344+3433, J0405+3803, 3C 454.3 \\
\hline BK131B & 18/06/06 & J0440-4333, J1159+0112, 3C 286, J0539-2839, J2344+3433, 3C 454.3 \\
\hline BH135G & 03/07/06 & J1150-0024, 3C 147, 3C 274 \\
\hline
\end{tabular}

studies of relatively faint radio sources (using suitable in-beam calibrators) should be a productive way of characterising the low-frequency radio sky at the highest resolution.

All these developments, emphasise the need to establish a suitable list of compact radio sources that may be good calibrators or reasonable commissioning targets for LOFAR and the RadioAstron mission. In this paper, we present the results of an automatic analysis of $327 \mathrm{MHz}$ data publicly available via the NRAO Very Long Baseline Array (VLBA), observed during the period 1 January 2003-31 December $2006^{2}$. In Sect. 2 we discuss the automatic pipeline procedure, and in Sect. 3 we present the main results of our analysis, including images of the individual sources. Notes and brief descriptions of some interesting sources are presented as a separate appendix. Final conclusions are also drawn in Sect. 3.

\section{Data analysis}

All publicly available VLBA $327 \mathrm{MHz}$ data, observed during the period 1 January 2003-31 December 2006 were downloaded from the archive and processed by an automatic pipeline implemented in NRAO's AIPS. 23 individual projects were processed (not including many multi-epoch sub-projects), comprising a total of 44 unique radio sources. Table 1 lists the various projects, and the sources observed. 8 of the 23 projects included multiplepass correlation (see Table 1) to target multiple sources in the same field of view. Table 2 gives details of the individual sources. The sources in the sample are located fairly randomly on the northern sky, with a range of declinations from $\delta=-33$ degrees to $\delta=+77$ degrees. The pipeline was designed to amplitude calibrate the data and fringe fit each source individually, using a solution interval of $3 \mathrm{~min}$ in all cases. The data were averaged in time and frequency, after applying the initial calibration. The pipeline also produced preliminary images of each amplitude

\footnotetext{
2 The National Radio Astronomy Observatory is a facility of the National Science Foundation operated under cooperative agreement by Associated Universities, Inc.
}

calibrated and fringe-fitted (detected) source. For those sources detected, manual editing and imaging was conducted using the AIPS tasks CALIB and IMAGR.

Of the 44 sources, 34 were detected on all or some subset of VLBA baselines (Table 3) and 10 sources were not detected, on any VLBA baseline: J0440-4333, J0959+6932 (M 82), J0955+6940; J1159+0112, J1230-1139, J2007+4029, $\mathrm{J} 2322+0812$, J2236+2828, J2334+0736 and J2344+3433. 30 were successfully imaged and 4 sources were either severely resolved or did not have enough data (or UV-coverage) to be successfully imaged. One special case is Cygnus-A. This was barely detected $(9 \sigma)$ only on the shortest VLBA baseline, and only by introducing an offset point model. For complex sources like Cygnus-A, a more complex model within FRING might result in more detections.

\section{Results and discussion}

Table 3 lists the sources that were detected. Note that most sources were observed by more than 1 project (see Table 1). In these cases, the "best" observation was selected, based on quality and quantity of the data. Figures 1-5 show the contour plots of the sources ${ }^{3}$. Given that the longest LOFAR baseline at $240 \mathrm{MHz}$, corresponds to $2 \mathrm{M} \lambda$, all sources that are detected on all VLBA baselines are also likely to be detected by LOFAR. All of the 30 sources imaged, are compact enough to be detected on baselines greater than $2 \mathrm{M} \lambda$, and many show extended structures and are resolved even beyond this baseline length (e.g. see 3C 84, Fig. 1). However, for LOFAR, many of these sources will remain unresolved (see the amplitude vs. UV-distance plots presented in Figs. 1-8). Many of the sources imaged in our programme should therefore make excellent LOFAR primary phase/bandpass calibrators, especially on the longest LOFAR baselines. Table 4 lists the best candidate calibrators.

\footnotetext{
3 The corresponding plots of flux density against UV-distance and U vs. V plots, will be available on the electronic version.
} 
Table 2. General properties of the 44 radio sources detected by VLBA at $92 \mathrm{~cm}$ from 2003-2006. Obtained from The NASA Extragalactic Database.

\begin{tabular}{|c|c|c|c|c|c|}
\hline IAU J2000 Source Name & Other Aliases & Type & Redshift & RA (J2000) & Dec (J2000) \\
\hline $\mathrm{J} 0204+3649$ & $0201+365$ & $\mathrm{Q}$ & 2.912000 & $02 \mathrm{~h} 04 \mathrm{~m} 55.596 \mathrm{~s}$ & $+36 \mathrm{~d} 49 \mathrm{~m} 17.996 \mathrm{~s}$ \\
\hline J0319+4130 & 3C 84 & G & 0.017559 & 03h19m48.160s & $+41 \mathrm{~d} 30 \mathrm{~m} 42.103 \mathrm{~s}$ \\
\hline $\mathrm{J} 0405+3803$ & $4 C+37.11$ & G & 0.055000 & $04 \mathrm{~h} 05 \mathrm{~m} 49.262 \mathrm{~s}$ & $+38 \mathrm{~d} 03 \mathrm{~m} 32.236 \mathrm{~s}$ \\
\hline J0407-3303 & $0405-331$ & $\mathrm{Q}$ & 2.562000 & $04 \mathrm{~h} 07 \mathrm{~m} 33.914 \mathrm{~s}$ & $-33 \mathrm{~d} 03 \mathrm{~m} 46.359 \mathrm{~s}$ \\
\hline J0418+3801 & 3C 111 & $\mathrm{G}$ & 0.048500 & $04 \mathrm{~h} 18 \mathrm{~m} 21.277 \mathrm{~s}$ & $+38 \mathrm{~d} 01 \mathrm{~m} 35.900 \mathrm{~s}$ \\
\hline J0423-0120 & - & $\mathrm{Q}$ & 0.914000 & $04 \mathrm{~h} 23 \mathrm{~m} 15.801 \mathrm{~s}$ & $-01 \mathrm{~d} 20 \mathrm{~m} 33.065 \mathrm{~s}$ \\
\hline $\mathrm{J} 0433+0521$ & $3 \mathrm{C} 120$ & G & 0.033100 & $04 \mathrm{~h} 33 \mathrm{~m} 11.096 \mathrm{~s}$ & $+05 \mathrm{~d} 21 \mathrm{~m} 15.619 \mathrm{~s}$ \\
\hline J0440-4333 & $0438-436$ & Q & 2.863000 & 04h40m17.180s & $-43 \mathrm{~d} 33 \mathrm{~m} 08.604 \mathrm{~s}$ \\
\hline J0542+4951 & 3C 147 & Q & 0.545000 & $05 \mathrm{~h} 42 \mathrm{~m} 36.138 \mathrm{~s}$ & $+49 \mathrm{~d} 51 \mathrm{~m} 07.234 \mathrm{~s}$ \\
\hline J0539-2839 & $0537-286$ & $\mathrm{Q}$ & 3.104000 & $05 \mathrm{~h} 39 \mathrm{~m} 54.281 \mathrm{~s}$ & $-28 \mathrm{~d} 39 \mathrm{~m} 55.948 \mathrm{~s}$ \\
\hline J0959+6932 & M 82 & G & 0.000677 & $09 \mathrm{~h} 59 \mathrm{~m} 10.639 \mathrm{~s}$ & $+69 \mathrm{~d} 32 \mathrm{~m} 17.724 \mathrm{~s}$ \\
\hline J0955+6940 & - & - & - & 09h59m55.694 & $+69 \mathrm{~d} 40 \mathrm{~m} 43.690 \mathrm{~s}$ \\
\hline $\mathrm{J} 1150-0024$ & $1148-001$ & Q & 1.976200 & $11 \mathrm{~h} 50 \mathrm{~m} 43.871 \mathrm{~s}$ & $-00 \mathrm{~d} 23 \mathrm{~m} 54.205 \mathrm{~s}$ \\
\hline $\mathrm{J} 1230+1223$ & 3С 274, M 87 & $\mathrm{G}$ & 0.004360 & $12 \mathrm{~h} 30 \mathrm{~m} 49.423 \mathrm{~s}$ & $+12 \mathrm{~d} 23 \mathrm{~m} 28.044 \mathrm{~s}$ \\
\hline $\mathrm{J} 1159+0112$ & $1157+014$ & Q & 1.999650 & $11 \mathrm{~h} 59 \mathrm{~m} 44.829 \mathrm{~s}$ & $+01 \mathrm{~d} 12 \mathrm{~m} 06.960 \mathrm{~s}$ \\
\hline $\mathrm{J} 1229+0203$ & $3 \mathrm{C} 273$ & $\mathrm{Q}$ & 0.158339 & $12 \mathrm{~h} 29 \mathrm{~m} 06.700 \mathrm{~s}$ & $+02 \mathrm{~d} 03 \mathrm{~m} 08.598 \mathrm{~s}$ \\
\hline J1230-1139 & $1228-113$ & $\mathrm{Q}$ & 3.528000 & $12 \mathrm{~h} 30 \mathrm{~m} 55.556 \mathrm{~s}$ & $-11 \mathrm{~d} 39 \mathrm{~m} 09.796 \mathrm{~s}$ \\
\hline $\mathrm{J} 1243+1622$ & 3C 275.1 & $\mathrm{Q}$ & 0.555100 & $12 \mathrm{~h} 43 \mathrm{~m} 57.657 \mathrm{~s}$ & $+16 \mathrm{~d} 22 \mathrm{~m} 53.440 \mathrm{~s}$ \\
\hline $\mathrm{J} 1331+3030$ & 3C 286 & G & 0.586000 & $13 \mathrm{~h} 31 \mathrm{~m} 08.288 \mathrm{~s}$ & $+30 \mathrm{~d} 30 \mathrm{~m} 32.959 \mathrm{~s}$ \\
\hline $\mathrm{J} 1333+1649$ & $1331+170$ & Q & 2.084000 & $13 \mathrm{~h} 33 \mathrm{~m} 35.783 \mathrm{~s}$ & $+16 \mathrm{~d} 49 \mathrm{~m} 04.015 \mathrm{~s}$ \\
\hline $\mathrm{J} 1512-0905$ & $1510-089$ & $\mathrm{Q}$ & 0.360000 & $15 \mathrm{~h} 12 \mathrm{~m} 50.533 \mathrm{~s}$ & $-09 \mathrm{~d} 05 \mathrm{~m} 59.829 \mathrm{~s}$ \\
\hline J1619+2247 & - & Q & 1.987000 & $16 \mathrm{~h} 19 \mathrm{~m} 14.825 \mathrm{~s}$ & $+22 \mathrm{~d} 47 \mathrm{~m} 47.85 \mathrm{~s}$ \\
\hline J1624+2345 & 3C 336 & Q & 0.927398 & $16 \mathrm{~h} 24 \mathrm{~m} 39.088 \mathrm{~s}$ & $+23 \mathrm{~d} 45 \mathrm{~m} 12.240 \mathrm{~s}$ \\
\hline $\mathrm{J} 1642+3948$ & $3 \mathrm{C} 345$ & $\mathrm{Q}$ & 0.592800 & $16 \mathrm{~h} 42 \mathrm{~m} 58.810 \mathrm{~s}$ & $+39 \mathrm{~d} 48 \mathrm{~m} 36.994 \mathrm{~s}$ \\
\hline $\mathrm{J} 1746+6226$ & $4 C+62.29$ & Q & 3.889000 & $17 \mathrm{~h} 46 \mathrm{~m} 14.034 \mathrm{~s}$ & $+62 \mathrm{~d} 26 \mathrm{~m} 54.738 \mathrm{~s}$ \\
\hline J1816+3457 & - & G & 0.244800 & $18 \mathrm{~h} 16 \mathrm{~m} 23.901 \mathrm{~s}$ & $+34 \mathrm{~d} 57 \mathrm{~m} 45.748 \mathrm{~s}$ \\
\hline J1821+3945 & - & RS & - & $18 \mathrm{~h} 21 \mathrm{~m} 59.701 \mathrm{~s}$ & $+39 \mathrm{~d} 45 \mathrm{~m} 59.657 \mathrm{~s}$ \\
\hline J1829+4844 & $3 \mathrm{C} 380$ & Q & 0.692000 & $18 \mathrm{~h} 29 \mathrm{~m} 31.739 \mathrm{~s}$ & $+48 \mathrm{~d} 44 \mathrm{~m} 46.971 \mathrm{~s}$ \\
\hline $\mathrm{J} 1902+3159$ & 3C 395 & Q & 0.635000 & $19 \mathrm{~h} 02 \mathrm{~m} 55.939 \mathrm{~s}$ & $+31 \mathrm{~d} 59 \mathrm{~m} 41.702 \mathrm{~s}$ \\
\hline J1924+3329 & $4 C+33.48$ & RS & - & $19 \mathrm{~h} 24 \mathrm{~m} 17.476 \mathrm{~s}$ & $+33 \mathrm{~d} 29 \mathrm{~m} 29.484 \mathrm{~s}$ \\
\hline J1959+4044 & Cygnus-A & Q & 0.056075 & $19 \mathrm{~h} 59 \mathrm{~m} 28.357 \mathrm{~s}$ & $+40 \mathrm{~d} 44 \mathrm{~m} 02.097 \mathrm{~s}$ \\
\hline $\mathrm{J} 2005+7752$ & $2007+777$ & Q & 0.342000 & $20 \mathrm{~h} 05 \mathrm{~m} 30.999 \mathrm{~s}$ & $+77 \mathrm{~d} 52 \mathrm{~m} 43.248 \mathrm{~s}$ \\
\hline $\mathrm{J} 2007+4029$ & $2005+403$ & Q & 1.736000 & $20 \mathrm{~h} 07 \mathrm{~m} 44.945 \mathrm{~s}$ & $+40 \mathrm{~d} 29 \mathrm{~m} 48.604 \mathrm{~s}$ \\
\hline $\mathrm{J} 2022+6136$ & - & G & 0.227000 & $20 \mathrm{~h} 22 \mathrm{~m} 06.682 \mathrm{~s}$ & $+61 \mathrm{~d} 36 \mathrm{~m} 58.805 \mathrm{~s}$ \\
\hline $\mathrm{J} 2038+5119$ & 3C 418 & Q & 1.686000 & $20 \mathrm{~h} 38 \mathrm{~m} 37.035 \mathrm{~s}$ & $+51 \mathrm{~d} 19 \mathrm{~m} 12.663 \mathrm{~s}$ \\
\hline $\mathrm{J} 2202+4216$ & BL Lac & Q & 0.068600 & $22 \mathrm{~h} 02 \mathrm{~m} 43.291 \mathrm{~s}$ & $+42 \mathrm{~d} 16 \mathrm{~m} 39.980 \mathrm{~s}$ \\
\hline $\mathrm{J} 2253+1608$ & 3C 454.3 & Q & 0.859000 & $22 \mathrm{~h} 53 \mathrm{~m} 57.748 \mathrm{~s}$ & $+16 \mathrm{~d} 08 \mathrm{~m} 53.561 \mathrm{~s}$ \\
\hline $\mathrm{J} 2322+0812$ & $2320+079$ & Q & 2.090000 & $23 \mathrm{~h} 22 \mathrm{~m} 36.089 \mathrm{~s}$ & $+08 \mathrm{~d} 12 \mathrm{~m} 01.593 \mathrm{~s}$ \\
\hline $\mathrm{J} 2327+0846$ & NGC 7674 & G & 0.028924 & $23 \mathrm{~h} 27 \mathrm{~m} 56.710 \mathrm{~s}$ & $+08 \mathrm{~d} 46 \mathrm{~m} 44.135 \mathrm{~s}$ \\
\hline $\mathrm{J} 2327+0940$ & - & Q & 1.843000 & $23 \mathrm{~h} 27 \mathrm{~m} 33.581 \mathrm{~s}$ & $+09 \mathrm{~d} 40 \mathrm{~m} 09.463 \mathrm{~s}$ \\
\hline $\mathrm{J} 2330+1100$ & - & Q & 1.489000 & $23 \mathrm{~h} 30 \mathrm{~m} 40.852 \mathrm{~s}$ & $+11 \mathrm{~d} 00 \mathrm{~m} 18.710 \mathrm{~s}$ \\
\hline $\mathrm{J} 2334+0736$ & - & Q & 0401000 & $23 \mathrm{~h} 34 \mathrm{~m} 12.828 \mathrm{~s}$ & $+07 \mathrm{~d} 36 \mathrm{~m} 27.552 \mathrm{~s}$ \\
\hline $\mathrm{J} 2336+2828$ & - & RS & - & $23 \mathrm{~h} 36 \mathrm{~m} 22.471 \mathrm{~s}$ & $+28 \mathrm{~d} 28 \mathrm{~m} 57.413 \mathrm{~s}$ \\
\hline $\mathrm{J} 2344+3433$ & $2342+342$ & Q & 3.053000 & $23 \mathrm{~h} 44 \mathrm{~m} 51.254 \mathrm{~s}$ & $+34 \mathrm{~d} 33 \mathrm{~m} 48.640 \mathrm{~s}$ \\
\hline
\end{tabular}

Of the 30 imaged sources, only 13 sources have been detected on the very longest VLBA baselines (9-9.5 M $\lambda$ ). These 13 sources may prove to be interesting targets for RadioAstron. In particular, sources such as 3C 84, 1148-001, J2330+11, 1510089, 0537-286 and 3C 345 may be employed as "in-beam" calibrators for deep wide-field $327 \mathrm{MHz}$ ground based VLBI observations. These sources are strong enough to be well detected by ground based VLBI arrays, but not so bright as to significantly raise the system noise of the antennas or introduce potential dynamic range limitations in the image plane.

It is interesting to note how the morphology of some of the sources in our sample change with baseline length. A good example is Cygnus-A. The first observations taken with the LOFAR test station at Exloo (1 km baseline) show that Cygnus$\mathrm{A}$ is one of the brightest objects in the sky. However, the VLBA observations at $327 \mathrm{MHz}$ do not detect Cygnus-A on all but the shortest $(240 \mathrm{~km})$ baseline (using an offset point source model centred on one of the lobes, see the appendix for further discussion). We conclude that Cygnus-A will also be heavily resolved for LOFAR on the longest international baselines, at least at the highest observing frequencies. In particular, there is no guarantee that sources that make good calibrators for the LOFAR core, will also be appropriate for longer baseline lengths. Other bright sources will no-doubt show similar properties, although we also note that some of these bright sources (e.g. 3C 274) remain bright and compact on milliarcsecond scales.

More systematic surveys by the VLBA at $327 \mathrm{MHz}$ would be useful to further extend the list of potential calibrators and targets for both LOFAR and RadioAstron.

\section{Appendix A: Individual source comments}

In this appendix the properties of some of the more interesting sources in our sample are discussed. 
Table 3. List of 34 detected sources. The images of most of these sources are shown in Figs. 1-5.

\begin{tabular}{|c|c|c|c|c|}
\hline Source & Detected Baseline $(\mathrm{M} \lambda)$ & Imaged $(\mathrm{Y} / \mathrm{N})$ & Peak Flux Density (Jy/beam) & Total Flux Density, $S_{327}(\mathrm{Jy})$ \\
\hline J0204+3649 & 8.0 & $\mathrm{Y}$ & 0.337 & 0.481 \\
\hline J0319+4130 (3C 84) & 9.0 & Y & 2.289 & 4.625 \\
\hline J0405+3803 & 4.0 & $\mathrm{Y}$ & 0.427 & 0.539 \\
\hline J0407-3303 & 9.5 & $\mathrm{Y}$ & 0.200 & 0.225 \\
\hline J0418+3801 (3C 111) & 6.0 & $\mathrm{Y}$ & 0.328 & 0.533 \\
\hline J0423-0120 & 9.5 & $\mathrm{Y}$ & 0.869 & 1.099 \\
\hline $\mathrm{J} 0433+0521$ (3C 120) & 9.5 & $\mathrm{Y}$ & 1.263 & 1.771 \\
\hline J0542+4951 (3C 147) & 8.0 & $\mathrm{Y}$ & 3.850 & 40.980 \\
\hline J0539-2839 & 9.5 & $\mathrm{Y}$ & 0.498 & 0.564 \\
\hline J1150-0024 & 9.5 & $\mathrm{Y}$ & 2.645 & 3.403 \\
\hline $\mathrm{J} 1230+1223$ (3C 274) & 9.5 & $\mathrm{Y}$ & 1.199 & 2.154 \\
\hline $\mathrm{J} 1229+0203$ (3C 273) & 9.5 & $\mathrm{Y}$ & 4.028 & 5.23 \\
\hline $\mathrm{J} 1243+1622$ (3C 275.1) & 2.0 & $\mathrm{Y}$ & 0.422 & 1.413 \\
\hline $\mathrm{J} 1331+3030$ (3C 286) & 9.5 & $\mathrm{Y}$ & 6.289 & 16.926 \\
\hline $\mathrm{J} 1333+1649$ & 6.0 & $\mathrm{Y}$ & 0.043 & 0.474 \\
\hline J1512-0905 & 9.5 & $\mathrm{Y}$ & 0.480 & 0.611 \\
\hline $\mathrm{J} 1619+2247$ & 2.5 & $\mathrm{~N}$ & - & - \\
\hline J1624+2345 (3C 336) & 4.5 & $\mathrm{~N}$ & - & - \\
\hline J1642+3948 (3C 345) & 9.5 & $\mathrm{Y}$ & 2.952 & 3.492 \\
\hline $\mathrm{J} 1746+6226$ & 6.5 & $\mathrm{Y}$ & 0.038 & 0.131 \\
\hline J1816+3457 & 6.0 & $\mathrm{Y}$ & 0.440 & 0.729 \\
\hline $\mathrm{J} 1821+3945$ & 5.0 & $\mathrm{Y}$ & 1.748 & 3.974 \\
\hline J1829+4844 & 8.0 & $\mathrm{Y}$ & 2.948 & 3.880 \\
\hline J1902+3159 (3C 395) & 5.0 & $\mathrm{Y}$ & 0.842 & 2.100 \\
\hline J1924+3329 & 2.0 & $\mathrm{Y}$ & 0.826 & 0.940 \\
\hline J1959+4044 (Cygnus-A) & 0.3 & $\mathrm{~N}$ & - & - \\
\hline $\mathrm{J} 2005+7752$ & 6.0 & $\mathrm{Y}$ & 0.508 & 0.602 \\
\hline $\mathrm{J} 2022+6136$ & 9.5 & $\mathrm{Y}$ & 0.635 & 0.990 \\
\hline $\mathrm{J} 2038+5119$ (3C 418) & 4.0 & $\mathrm{Y}$ & 1.234 & 3.320 \\
\hline J2202+4216 (BL Lac) & 6.0 & $\mathrm{Y}$ & 0.938 & 1.798 \\
\hline $\mathrm{J} 2253+1608(3 \mathrm{C} 454.3)$ & 9.0 & $\mathrm{Y}$ & 5.300 & 8.659 \\
\hline J2327+0846 (N7674) & 1.8 & $\mathrm{~N}$ & - & - \\
\hline $\mathrm{J} 2327+0940$ & 7.0 & $\mathrm{Y}$ & 0.317 & 0.396 \\
\hline $\mathrm{J} 2330+1100$ & 9.0 & $\mathrm{Y}$ & 0.774 & 0.844 \\
\hline
\end{tabular}

\section{$J 0405+3803$}

The data presented in this paper for J0405+3803, have previously been observed and published by Rodriguez et al. (2006). The images of $0402+379$ produced in this article, compare well with those of Rodriguez et al. (2006), and are consistent with lower resolution VLA images taken at $1.4 \mathrm{GHz}$ (Xu et al. 1995).

\section{J0433+0521 (3C 120)}

The VLBA images at $327 \mathrm{MHz}$ presented here are consistent with the images at $2.3 \mathrm{GHz}$ taken by Fey \& Charlot (2000), also using the VLBA. The results at $2.3 \mathrm{GHz}$ presents the core and jet of $3 \mathrm{C} 120$ with 3 jet components, all located within approximately 100 mas of the core. The results presented here show an extended jet that spans sin 300 mas.

\section{J0542+4951 (3C 147)}

3C 147 is very resolved at $327 \mathrm{MHz}$ and shows an elongated structure that spans $\sim 400$ milliarcsec, largely consistent with that observed by EVN-MERLIN at 1.6 GHz (Davis et al. 1996). The image is also in good agreement with higher resolution VLBA observations made at $8.5 \mathrm{GHz}$ and $2.3 \mathrm{GHz}$ (Fey \& Charlot 2000). We do not detect the emission seen by MERLIN to the North-East of the peak emission, that (Davis et al. 1996) interpret as evidence of outflow from a counter jet. There is also an extended structure to the South-East of the peak of the emission, which is also observed at $1.6 \mathrm{GHz}$ with EVNMERLIN and, 2.3/8.5 GHz (VLBA).

\section{J1230+1223 (M 87, 3C 274)}

The observations with the VLBA at $327 \mathrm{MHz}$ show that the wellknown jet extends up to $\sim 500$ mas $(0.5 \mathrm{arcsec})$ from the core. The superluminal component observed by the Hubble Space Telescope known as HST 1, (Biretta et al. 1999) is clearly detected, located approximately 0.8 arcsecs from the core. This has also recently been detected by the VLBA at $15 \mathrm{GHz}$ (Chang et al. 2008). At $15 \mathrm{GHz}$ HST-1 appears to be variable. When detected it has a flux density in the range of $14-22 \mathrm{mJy}$. At $327 \mathrm{MHz}$, we measure an integrated flux density of $317 \mathrm{mJy}$, implying a spectral index of $\alpha_{0.327}^{15} \sim-0.697$. The size of the component at 327 $\mathrm{MHz}$ is $48 \times 21$ milliarcsec in PA $\sim 144$ degrees.

\section{$J 1331+3030(3 C 286)$}

Our images of 3C 286 are consistent with other VLBA images at higher frequencies e.g. Fey \& Charlot (2000). The $327 \mathrm{MHz}$ observations are clearly extended in the same direction as that seen in the MERLIN images at $18 \mathrm{~cm}$ (Akujor et al. 1994). We do not see the counter jet report by (Akujor et al. 1994). 
PLot file version 1 created 31-OCT-2008 15:27:39
CONT: $0201+365$ IPOL $330.474 \mathrm{MHZ}$ 0201+365.ICL001.5

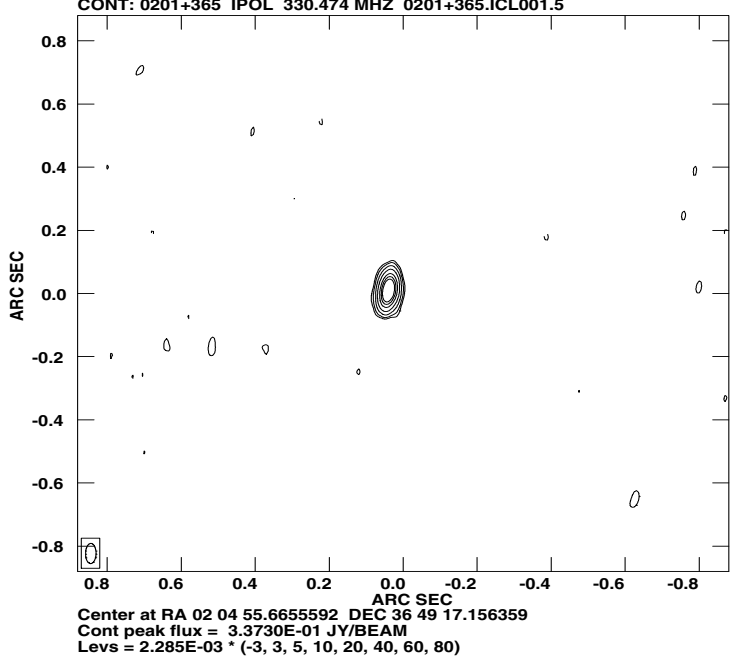

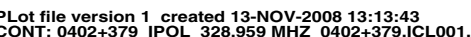
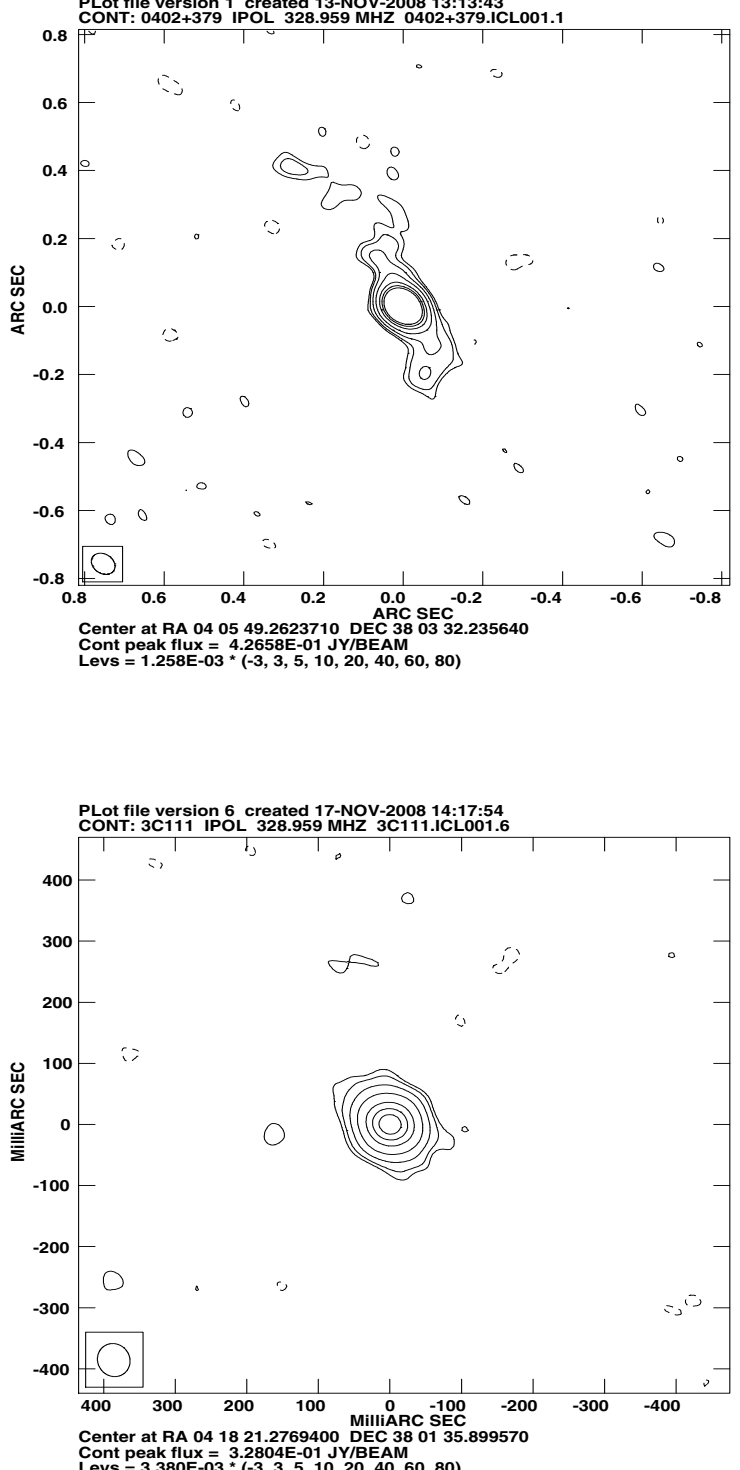

PLot file version 6 created 17-NOV-2008 14:14:54
CONT: 3C84 IPOL 328.959 MHZ 3C84.ICL01.4

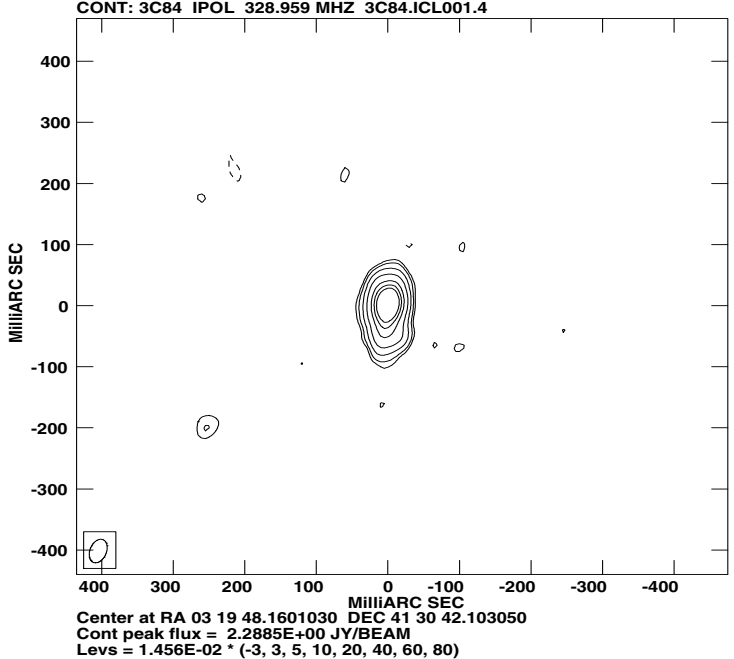

PLot file version 1 created 03-NOV-2008 12:12:32
CONT: 0405-331 IPOL 330.474 MHZ 0405-331.ICL001.12
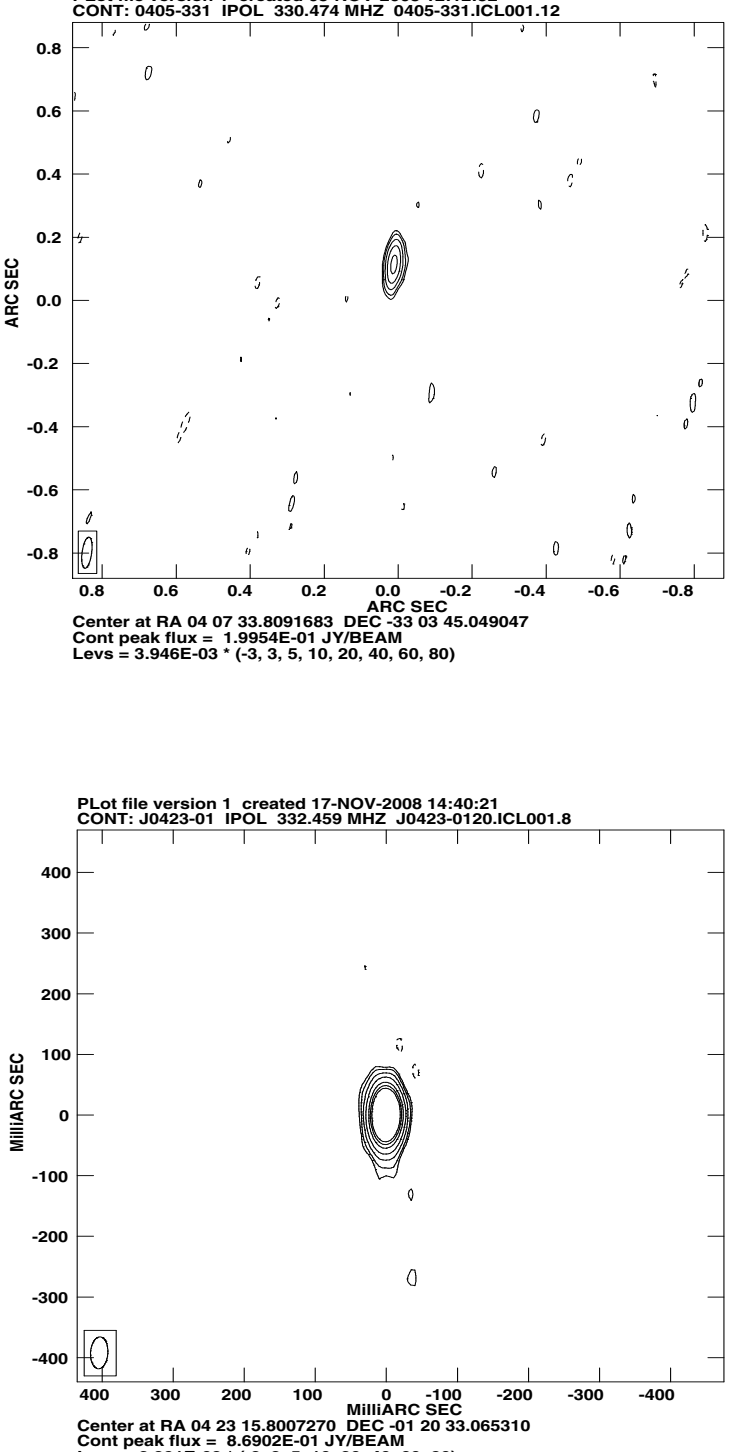

Fig. 1. Imaged sources at $327 \mathrm{MHz}$. The sources here in order are: J0204+3649, J0319+4130 (3C 84), J0405+3803, J0407-3303, J0418+3801 (3C 111), J0423-0120. 


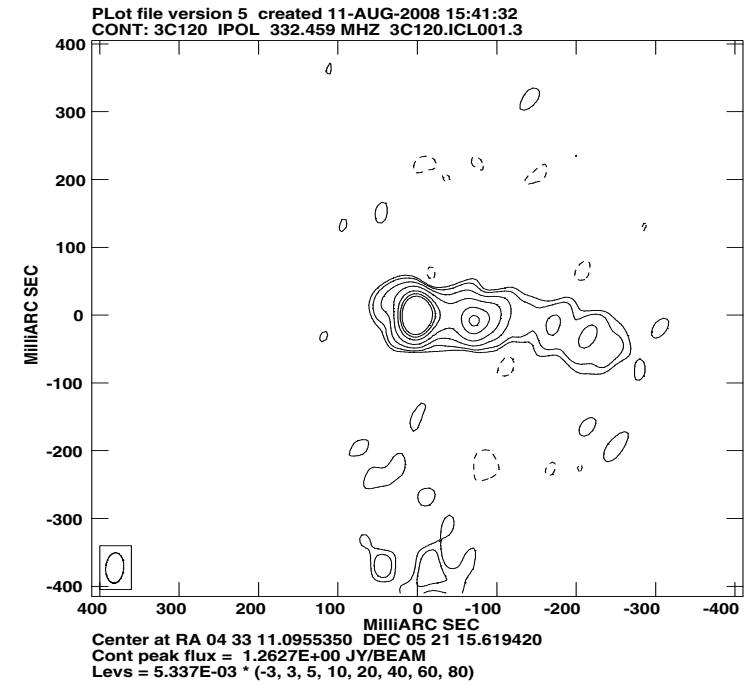

PLot file version 5 created 11-AUG-2008 $18: 54: 42$
CONT: 3 C147 IPOL 328.482 MHZ 3 C147.1CLLO1.4
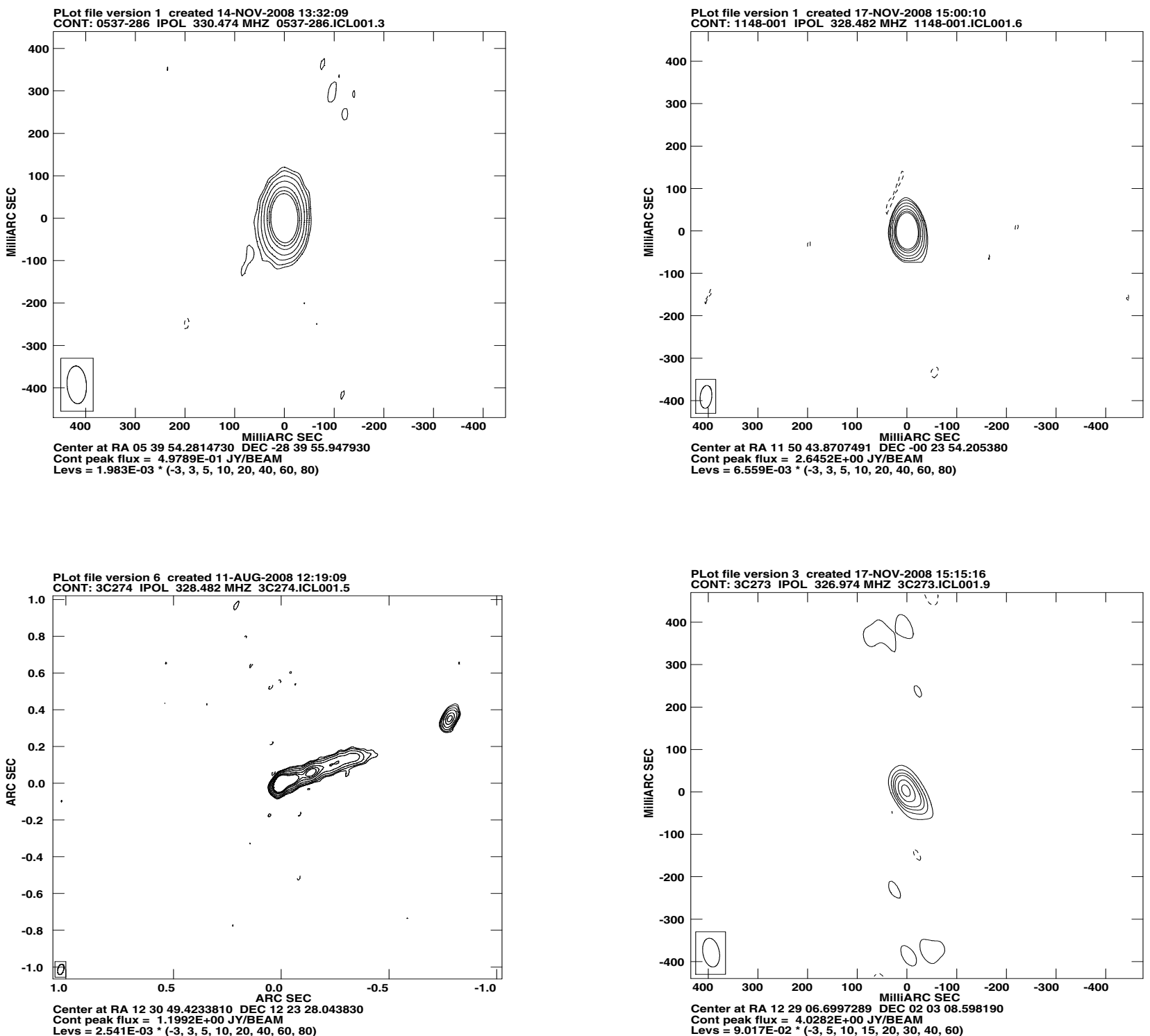

Fig. 2. Imaged sources at $327 \mathrm{MHz}$. The sources here in order are: J0433+0521 (3C 120), J0542+4951 (3C 147), J0539-2839, J1150-0024, $\mathrm{J} 1230+1223$ (3C 274), J1229+0203 (3C 273). 

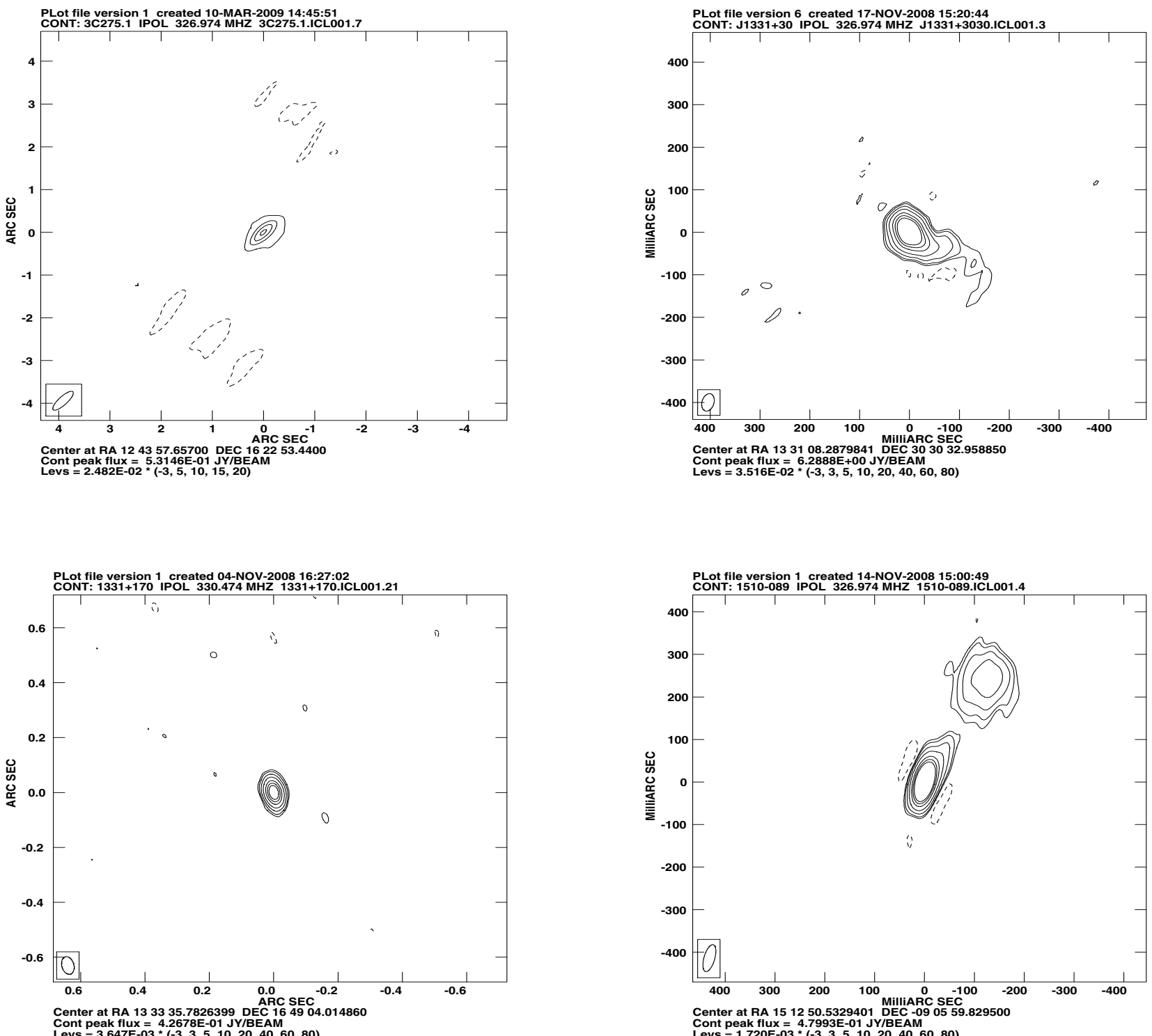

PLot file version 2 created 17-NOV-2008 15:38:38
CONT: 3 C345 IPOL 330.474 MHZ 3 C345.ICLO01.3
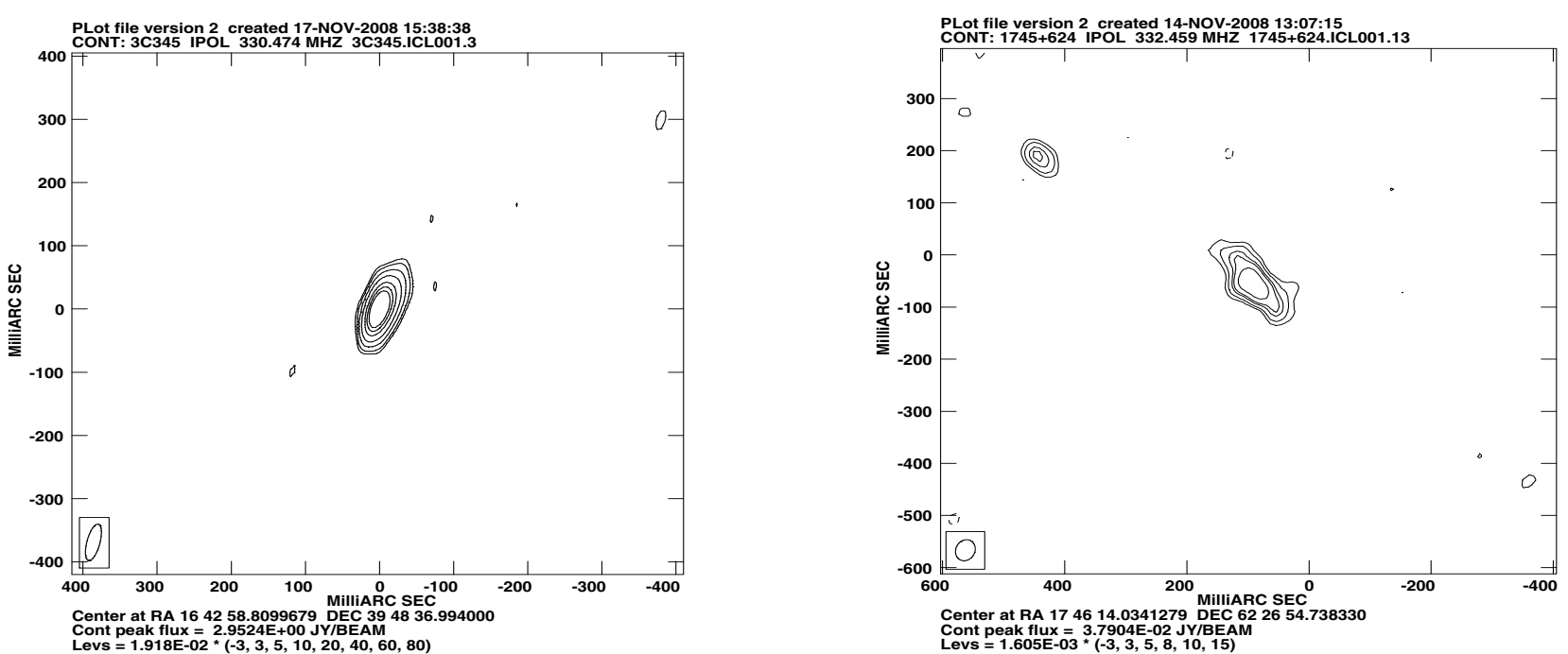

Fig. 3. Imaged sources at $327 \mathrm{MHz}$. The sources here in order are: J1243+1622 (3C 275.1), J1331+3030 (3C 286), J1333+1649, J1512-0905, $\mathrm{J} 1642+3948$ (3C 345), J1746+6226. 

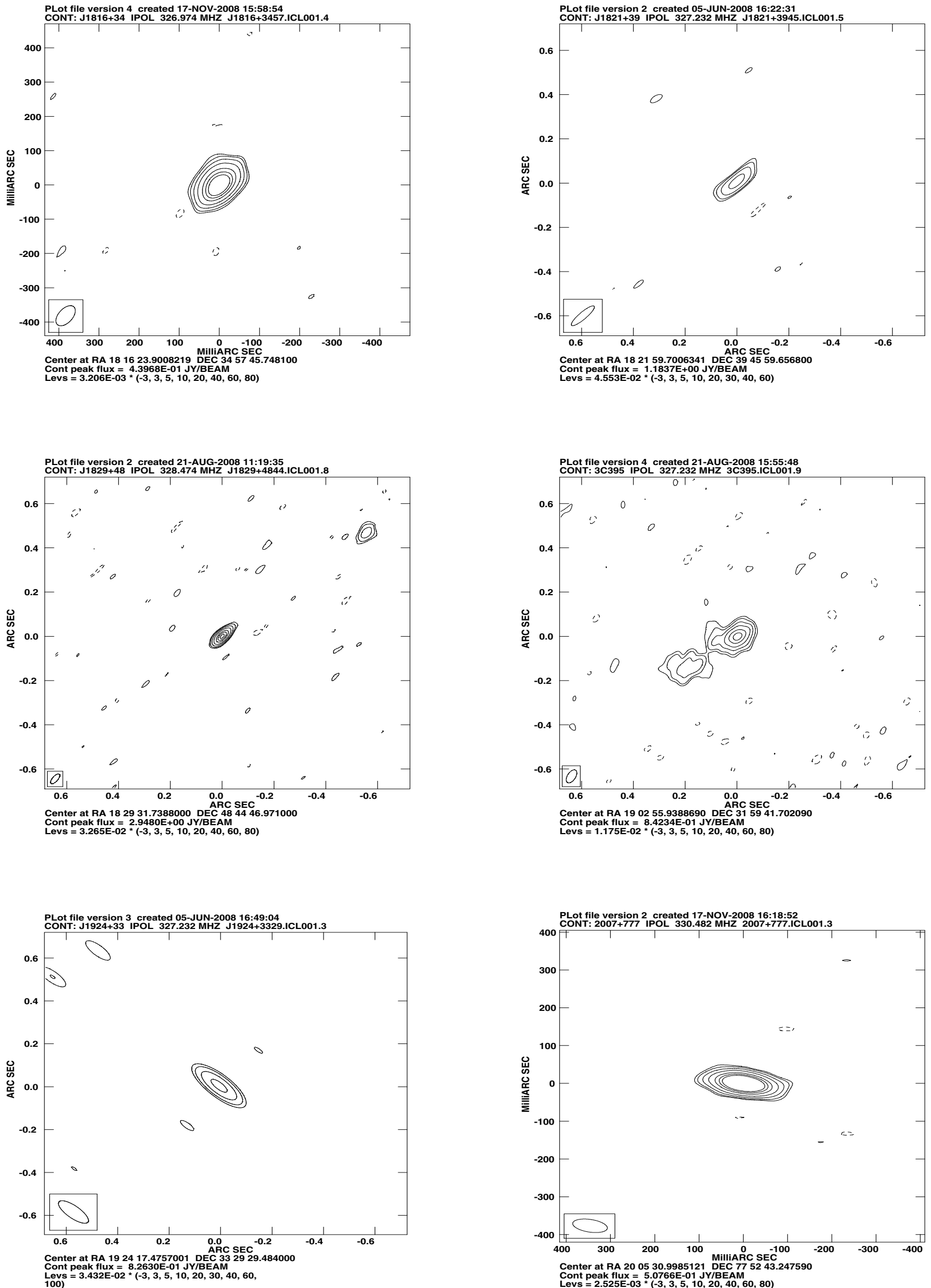

Fig. 4. Imaged sources at 327 MHz. The sources here in order are: J1816+3457, J1821+3945, J1829+4844, J1902+3159 (3C 395), J1924+3329, $\mathrm{J} 2005+7752$. 

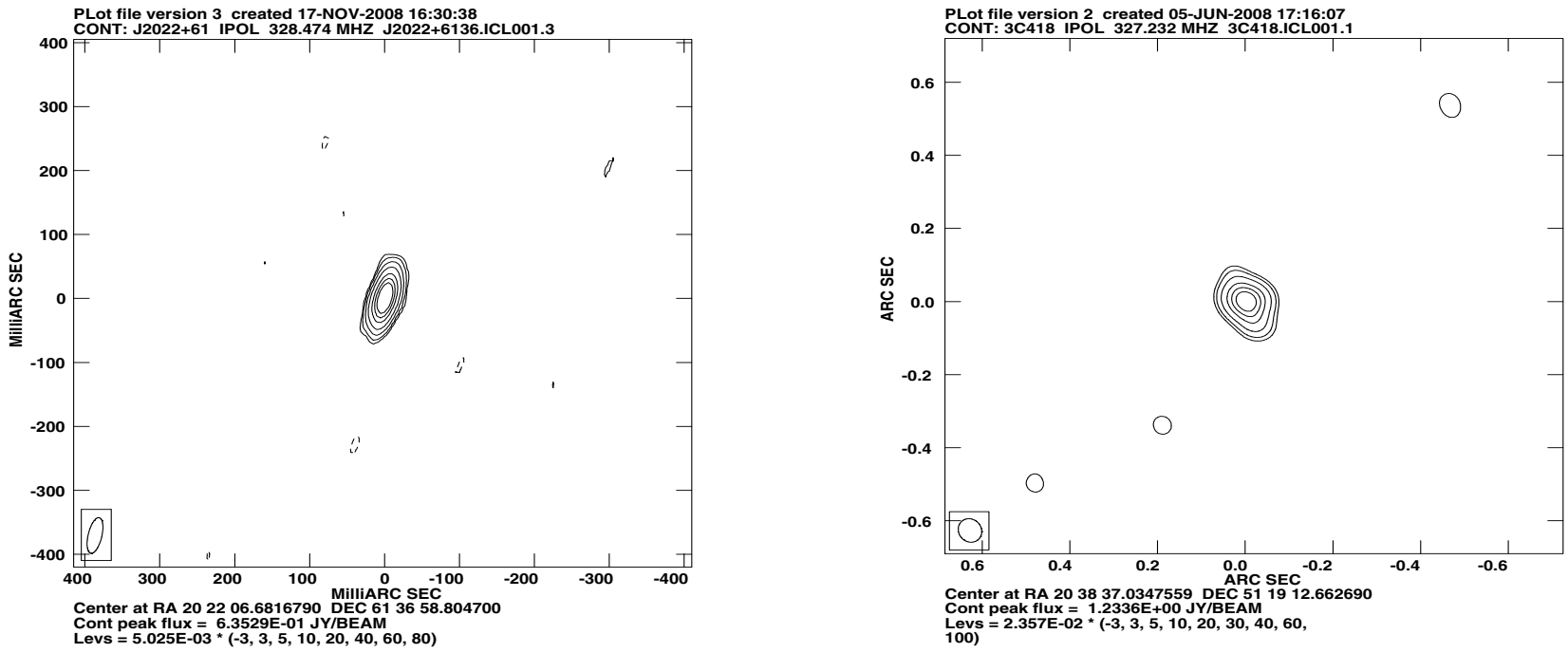

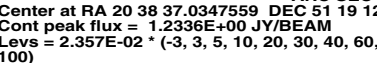

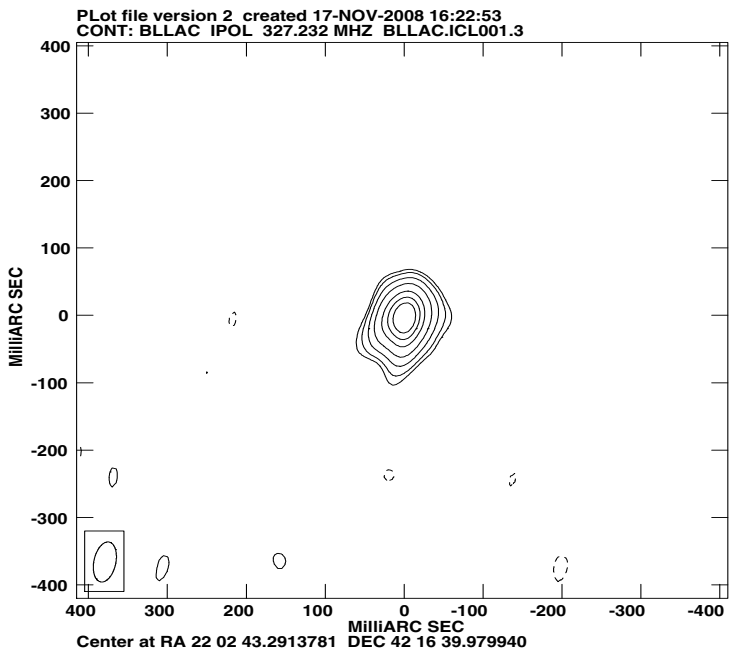

PLot file version 2 created 17-NOV-2008 16:26:56
CONT: 3C454.3 IPOL $330.982 \mathrm{MHZ} 3 \mathrm{C} 454.3 . \mathrm{CL}$

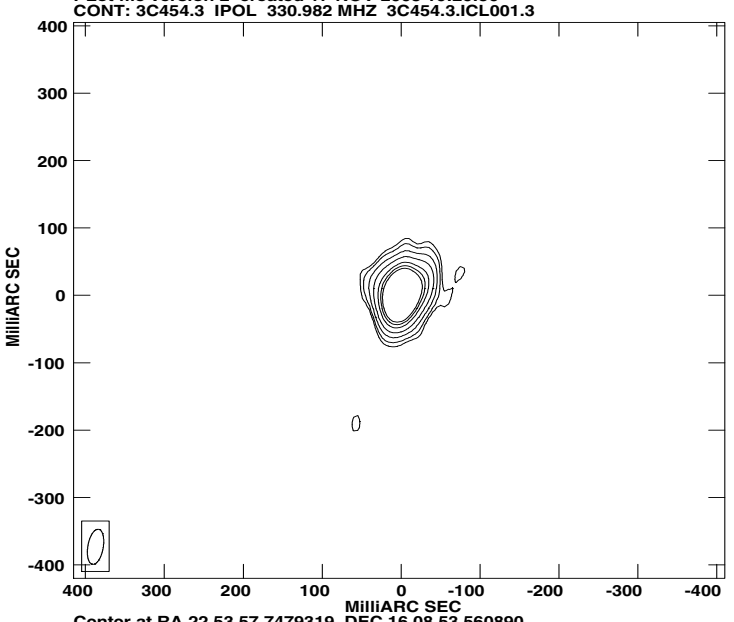

Center at RA 225357.7479319 DEC 160853.560890

Levs $=8.625 \mathrm{E}-03 \times(-3,3,5,10,20,40,60,80)$

Levs $=1.425 \mathrm{E}-02 *(-3,3,5,10,20,40,60,80)$

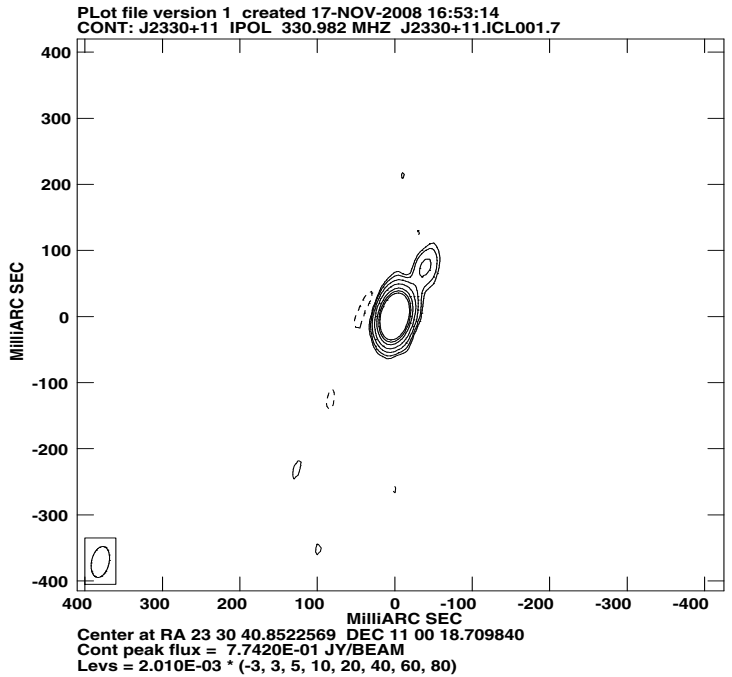

Fig. 5. Imaged sources at 327 MHz. The sources here in order are: J2022+6136, J2038+5119 (3C 418), J2202+4216 (BL Lac), J2253+1608 (3C 454.3), J2327+0940, J2330+11. 
Table 4. Best primary candidate calibrators for LOFAR on baselines $\sim 2 \mathrm{M} \lambda$.

\begin{tabular}{cccccc}
\hline \hline Source & Type & Redshift & RA & Dec & $S_{327}(\mathrm{Jy})$ \\
\hline J0319+4130 (3C 84) & Q & 0.017559 & $03 \mathrm{~h} 19 \mathrm{~m} 48.160 \mathrm{~s}$ & $+41 \mathrm{~d} 30 \mathrm{~m} 42.103 \mathrm{~s}$ & 4.625 \\
J0405+3803 & G & 0.055000 & $04 \mathrm{~h} 05 \mathrm{~m} 49.262 \mathrm{~s}$ & $+38 \mathrm{~d} 03 \mathrm{~m} 32.236 \mathrm{~s}$ & 0.539 \\
J0418+3801 (3C 111) & G & 0.048500 & $04 \mathrm{~h} 18 \mathrm{~m} 21.277 \mathrm{~s}$ & $+38 \mathrm{~d} 01 \mathrm{~m} 35.900 \mathrm{~s}$ & 0.533 \\
J0423-0120 & Q & 0.914000 & $04 \mathrm{~h} 23 \mathrm{~m} 15.801 \mathrm{~s}$ & $-01 \mathrm{~d} 20 \mathrm{~m} 33.065 \mathrm{~s}$ & 1.099 \\
J0539-2839 & Q & 3.104000 & $05 \mathrm{~h} 39 \mathrm{~m} 54.281 \mathrm{~s}$ & $-28 \mathrm{~d} 39 \mathrm{~m} 55.948 \mathrm{~s}$ & 0.564 \\
J1150-0024 & Q & 1.976200 & $11 \mathrm{~h} 50 \mathrm{~m} 43.871 \mathrm{~s}$ & $-00 \mathrm{~d} 23 \mathrm{~m} 54.205 \mathrm{~s}$ & 3.403 \\
J1642+3948 (3C 345) & Q & 0.592800 & $16 \mathrm{~h} 42 \mathrm{~m} 58.810 \mathrm{~s}$ & $+39 \mathrm{~d} 48 \mathrm{~m} 36.994 \mathrm{~s}$ & 3.492 \\
J1816+3457 & G & 0.244800 & $18 \mathrm{~h} 16 \mathrm{~m} 23.901 \mathrm{~s}$ & $+34 \mathrm{~d} 57 \mathrm{~m} 45.748 \mathrm{~s}$ & 0.729 \\
J2005+7752 & Q & 0.342000 & $20 \mathrm{~h} 05 \mathrm{~m} 30.999 \mathrm{~s}$ & $+77 \mathrm{~d} 52 \mathrm{~m} 43.248 \mathrm{~s}$ & 0.602 \\
J2022+6136 & G & 0.227000 & $20 \mathrm{~h} 22 \mathrm{~m} 06.682 \mathrm{~s}$ & $+61 \mathrm{~d} 36 \mathrm{~m} 58.805 \mathrm{~s}$ & 0.990 \\
J2202+4216 (BL Lac) & Q & 0.068600 & $22 \mathrm{~h} 02 \mathrm{~m} 43.291 \mathrm{~s}$ & $+42 \mathrm{~d} 16 \mathrm{~m} 39.980 \mathrm{~s}$ & 1.798 \\
J2253+1608 (3C 454.3) & Q & 0.859000 & $22 \mathrm{~h} 53 \mathrm{~m} 57.748 \mathrm{~s}$ & $+16 \mathrm{~d} 08 \mathrm{~m} 53.561 \mathrm{~s}$ & 8.659 \\
J2327+0940 & Q & 1.843000 & $23 \mathrm{~h} 27 \mathrm{~m} 33.581 \mathrm{~s}$ & $+09 \mathrm{~d} 40 \mathrm{~m} 09.463 \mathrm{~s}$ & 0.396 \\
J2330+1100 & Q & 1.489000 & $23 \mathrm{~h} 30 \mathrm{~m} 40.852 \mathrm{~s}$ & $+11 \mathrm{~d} 00 \mathrm{~m} 18.710 \mathrm{~s}$ & 0.844 \\
\hline
\end{tabular}

\section{J1512-0905}

The image presented here at $327 \mathrm{MHz}$ shows a jet component to the North-East of the core, which ends in a hot-spot. At higher frequencies $(2.3,8.6$ and $15 \mathrm{GHz})$, the hot-spot is not visible by the VLBA, but there is evidence for a jet component along the direction of the hot-spot (Fey \& Charlot 1997; Lister \& Homan 2005).

\section{$J 1746+6226(4 C+62.29)$}

At $327 \mathrm{MHz}$, we observe an extended core component with a morphology that is consistent with higher frequency VLBA observations at $2.3 \mathrm{GHz}$ and $8.6 \mathrm{GHz}$ Fey \& Charlot (2000). However, our images also reveal a counter jet with a hot-spot located approximately 0.2 arcsec to the North-East of the extended core. The PA of this counter jet feature is diametrically opposite to the higher frequency jet that is seen on the other side of the core.

\section{J1829+4844 (3C 380)}

The radio source $\mathrm{J} 1829+4844$ is identified with the very powerful steep spectrum radio source, 3C 380. Observations at decimetre and centimetre wavelengths have identified this object as an FR II source that is elongated along the line-of-sight (Wilkinson et al. 1991; Megn et al. 2006). This source has a very extended structure, with a number of compact components that corresponds to the core coincident with the optical quasar with a short (15 mas) jet, a hot-spot in the nearer lobe 0.7 arcsec from the core, and another slightly larger hot-spot 0.4 arcsec from the first (Megn et al. 2006). The lobes are located to the North-West of the core. Only the core and the hot-spot at 0.7 arcsec from the core are visible in the VLBA observations at $327 \mathrm{MHz}$.

\section{J1902+3159 (3C 395)}

3C 395 is identified with a 17 th magnitude quasar (Gelderman \& Whittle 1994), and has a complex radio structure on a wide range of angular scales. At $8.3 \mathrm{GHz}$ with the VLBA its corejet radio structure consists of two components (core and a hotspot) separated by approximately 15 mas, with the hot-spot located South-West of the core (Lara et al. 1997). VLBI images at $326 \mathrm{MHz}$, taken by Altschuler et al. (1995) show a compact and unresolved core, and an extended regio of emission separated by approximately 65 mas South-West of the core. Our results agree well with these low resolution images. There is also a fainter, extended component observed in our image at PA $111.7^{\circ}$. This was not observed by Altschuler et al. (1995).

\section{J1959+4044 (Cygnus-A)}

Despite being one of the brightest sources in the sky, Cygnus A was not detected by the VLBA at $327 \mathrm{MHz}$, except on the shortest baselines after including an offset point source model for the FRING task - offset in the direction of the South-Eastern lobe. Since it is located at low galactic latitude ( $b=5$ degrees), the core of Cygnus-A may be scatter broadened due to interstellar scattering in our own galaxy (Carilli et al. 1991). With a measured size of 1.6 mas at $5 \mathrm{GHz}$, we would expect the core size to be $\sim 400$ mas at $327 \mathrm{MHz}$. The core of Cygnus-A is therefore likely to be resolved by our observations. The same may also be true of hot-spots in the two lobe structures.

Acknowledgements. This research was supported by the EU Framework 6 Marie Curie Early Stage Training programme under contract number MEST-CT-200519669 "ESTRELA".

\section{References}

Akujor, C. E., Luedke, E., Browne, I. W. A., et al. 1994, A\&AS, 105, 247 Altschuler, D. R., Gurvits, L. I., Alef, W., et al. 1995, A\&AS, 114, 197 Biretta, J. A., Sparks, W. B., \& Macchetto, F. 1999, ApJ, 520, 621

Carilli, C. L., Bartel, N., \& Linfield, R. P. 1991, AJ, 102, 1691

Chang, C. S., Ros, E., Kovalev, Y. Y., \& Lister, M. L. 2008 [arXiv:0812.0126]

Chuprikov, A. A., Girin, I. A., Likhachev, S. F., et al. 1999, New Astron. Rev., 43, 747

Davis, R. J., Diamond, P. J., \& Goss, W. M. 1996, MNRAS, 283, 1105

Fey, A. L., \& Charlot, P. 1997, ApJS, 111, 95

Fey, A. L., \& Charlot, P. 2000, ApJS, 128, 17

Garrett, M. A., Rampadarath, H., Lenc, E., \& Wucknitz, O. 2009, [arXiv: 0902 .2534v2]

Gelderman, R., \& Whittle, M. 1994, ApJS, 91, 491

Lara, L., Muxlow, T. W. B., Alberdi, A., et al. 1997, A\&A, 319, 405

Lenc, E., Garrett, M. A., Wucknitz, O., Anderson, J. M., \& Tingay, S. J. 2008, ApJ, 673, 78

Lister, M. L., \& Homan, D. C. 2005, AJ, 130, 1389

Megn, A. V., Rashkovskii, S. L., Shepelev, V. A., et al. 2006, Astron. Rep., 50, 692

Rodriguez, C., Taylor, G. B., Zavala, R. T., et al. 2006, ApJ, 646, 49

Wilkinson, P. N., Akujor, C. E., Cornwell, T. J., \& Saikia, D. J. 1991, MNRAS, 248, 86

Xu, W., Readhead, A. C. S., Pearson, T. J., Polatidis, A. G., \& Wilkinson, P. N. 1995, ApJS, 99, 297

Zakharov, A. F. 2007, Serbian Astron. J., 174, 1 